Article

\title{
Genome Wide Identification of Respiratory Burst Oxidase Homolog (Rboh) Genes in Citrus sinensis and Functional Analysis of CsRbohD in Cold Tolerance
}

\author{
Yueliang Zhang ${ }^{1,+}$, Yiwu Zhang ${ }^{1,+}{ }^{,}$Li Luo ${ }^{1}$, Chunyi Lu ${ }^{1}$, Weiwen Kong ${ }^{1,2}$, Libao Cheng ${ }^{1,2}$, Xiaoyong Xu ${ }^{1,2, *(D)}$ \\ and Jihong $\operatorname{Liu}^{3, *(1)}$ \\ 1 School of Horticulture and Plant Protection, Yangzhou University, Yangzhou 225009, China; \\ 202000000071@just.edu.cn (Y.Z.); zyw_18796609849@163.com (Y.Z.); abcd17869359501@163.com (L.L.); \\ luaulu@163.com (C.L.); wwkong@yzu.edu.cn (W.K.); lbcheng@yzu.edu.cn (L.C.) \\ 2 Joint International Research Laboratory of Agriculture and Agri-Product Safety (The Ministry of Education), \\ Yangzhou University, Yangzhou 225009, China \\ 3 Key Laboratory of Horticultural Plant Biology (MOE), College of Horticulture and Forestry Science, \\ Huazhong Agricultural University, Wuhan 430070, China \\ * Correspondence: xyxu@yzu.edu.cn (X.X.); liujihong@mail.hzau.edu.cn (J.L.) \\ + These authors contributed equally to this work.
}

check for updates

Citation: Zhang, Y.; Zhang, Y.; Luo, L.; Lu, C.; Kong, W.; Cheng, L.; Xu, X.; Liu, J. Genome Wide Identification of Respiratory Burst Oxidase Homolog (Rboh) Genes in Citrus sinensis and Functional Analysis of CsRbohD in Cold Tolerance. Int. J. Mol. Sci. 2022, 23, 648. https://doi.org/10.3390/ ijms 23020648

Academic Editor: Zsófia Bánfalvi

Received: 2 December 2021

Accepted: 4 January 2022

Published: 7 January 2022

Publisher's Note: MDPI stays neutral with regard to jurisdictional claims in published maps and institutional affiliations.

Copyright: (C) 2022 by the authors. Licensee MDPI, Basel, Switzerland. This article is an open access article distributed under the terms and conditions of the Creative Commons Attribution (CC BY) license (https:// creativecommons.org/licenses/by/ $4.0 /)$.

\begin{abstract}
Respiratory burst oxidase homologs (Rbohs) are critical enzymes involved in the generation of reactive oxygen species (ROS) that play an important role in plant growth and development as well as various biotic and abiotic stresses in plants. Thus far, there have been few reports on the characterization of the Rboh gene family in Citrus. In this study, seven Rboh genes (CsRbohA CsRbohG) were identified in the Citrus sinensis genome. The CsRboh proteins were predicted to localize to the cell membrane. Most CsRbohs contained four conserved domains, an EF-hand domain, and a transmembrane region. Phylogenetic analysis demonstrated that the CsRbohs were divided into five groups, suggesting potential distinct functions and evolution. The expression profiles revealed that these seven CsRboh genes displayed tissue-specific expression patterns, and five CsRboh genes were responsive to cold stress. Fourteen putative cis-acting elements related to stress response, hormone response, and development regulation were present within the promoters of CsRboh genes. The insilico microRNA target transcript analyses indicated that CsRbohE might be targeted by csi-miR164. Further functional and physiological analyses showed that the knockdown of CsRbohD in trifoliate orange impaired resistance to cold stress. As a whole, our results provide valuable information for further functional studies of the CsRboh genes in response to cold stress.
\end{abstract}

Keywords: Citrus; respiratory burst oxidase homologs; cold stress; ROS

\section{Introduction}

Plant NADPH oxidases (NOXs), known as Rbohs and encoded by Rboh genes, are homologs of the mammalian phagocyte NOXs catalytic subunit gp91phox [1]. Rbohs often consist of six transmembrane central regions, two heme groups, cytosolic FAD- and NADPH-binding domains, and two EF-hand calcium-binding domains [2]. Through these domains, Rbohs can transfer electrons to extracellular $\mathrm{O}_{2}$ to produce $\mathrm{O}_{2}{ }^{-}$, and then rapidly converted into $\mathrm{H}_{2} \mathrm{O}_{2}$ by superoxide dismutase (SOD, EC 1.15.1.1). Increasing evidence suggests that Rboh-mediated ROS production is critical for plant growth and development as well as plant response to abiotic and biotic stresses [1,3,4]. Consequently, Rbohs are considered the important nodes in the ROS signaling network.

The first plant Rboh (OsRbohA) was identified in Oryza sativa [5], and more Rboh genes have been subsequently characterized in many other plant species. Rbohs are encoded by multiple genes, and the gene family has been identified and characterized in some plant species. So far, ten Rboh genes were found in Arabidopsis [6], nine in rice [7], seven 
in grapes [8], fourteen in Nicotiana tabacum [9], twenty-six in upland cotton [10], nine in apples [11], seven in strawberries [12], seven in jatropha [13], eight in cassava [14], fourteen in Brassica rapa [15], and eight in pepper [16]. Analysis of gene expression patterns may help to understand the possible function of Rboh family genes. In Arabidopsis, AtRbohA-G and $A t R b o h I$ were expressed in the roots, $A t R b o h D$ and $A t R b o h F$ in all tissues, and $A t R b o h H$ and AtRbohJ in pollen and stamen [6]. In rice, all Rboh genes, except for OsRbohD and OsRbohH, are constitutively expressed in roots, leaves, shoots, and calli [17]. In upland cotton, Ghrboh15 and Ghrboh16 were expressed constitutively in the stamen [10]. In pepper, CaRbohA, $\mathrm{CaRbohB}$, and $\mathrm{CaRbohD}$ genes were highly expressed in the five tissues, but $\mathrm{CaRbohC}, \mathrm{CaRbohF}$, and $\mathrm{CaRbohG}$ genes were weakly expressed [16]. This differential tissue expression of Rboh genes has also been widely described in other plants $[9,11,12,18]$, implying that the Rboh genes played important and diverse functional roles in different tissues.

Interestingly, Rboh genes are notably expressed under various abiotic stresses, especially low temperatures. For example, the transcription levels of FvRbohA and FvRbohD in strawberries were significantly induced by cold stress, followed by an enhancement in NADPH oxidase activity [12]. Five GmRboh genes $(A 2, C 1, F 1, G$, and H2) and four GmRboh genes $(B 1, B 2, D 2$, and $E 2)$ were up-regulated under cold stress in soybean roots and leaves, respectively [18]. The expression of seven NtabRboh genes $(A, B, C, M, N, O$, and $P)$ were increased markedly following cold treatment in Nicotiana tabacum leaves [9]. In pepper, cold treatment significantly enhanced the expression of CaRbohA and CaRbohB genes [16]. These results indicate that $R b o h$ genes are involved in cold stress responses, and the roles need to be further confirmed by transgenic studies in the future.

Citrus is one of the most important fruit crops planted worldwide, ranking first in production and yield among all fruit crops in 2019 (FAOSTAT data, https:/ /www.fao. org /faostat/en/ accessed on 3 September 2021). As one of the sessile organisms, Citrus is frequently subjected to various biotic or abiotic stresses, including cold stress [19]. Considering that Rbohs play key roles in response to cold stress, the identification of Citrus Rbohs' function offers an alternative way to enhance resistance to low-temperature stress. To the best of our knowledge, few efforts have been made to identify and characterize the Rboh gene family in Citrus [20]. In the current study, we identified the Rboh gene family members from the Citrus sinensis genome and systematically analyzed chromosomal localization, gene structures, conserved motifs, phylogenetic relationships, cis-acting elements, and potential microRNA (miRNA) target sites. Secondly, the expression profiles of CsRboh genes in different tissues and their response to cold stress were investigated by RNA-Seq data and quantitative real-time PCR (qRT-PCR) analysis, respectively. Finally, we investigated the function of CsRbohD associated with cold stress using virus-induced gene silencing (VIGS) system. As a whole, our results provide valuable information for further functional analysis of CsRboh genes in response to cold stress.

\section{Results}

\subsection{Identification of Rboh Gene Family Members in C. sinensis}

A total of seven putative CsRboh genes were identified from the C. sinensis genome by HMMER and BLASTP searches. These candidate CsRboh genes were named as CsRbohA-G based on their localization in the $C$. sinensis genome and the widely accepted nomenclature system. CsRboh genes were localized on chromosomes three (CsRbohA), four (CsRbohB), five (CsRbohC and CsRbohD), seven (CsRbohE), and eight (CsRbohF and CsRbohG) (Figure 1). As shown in Table 1, the predicted CsRboh proteins consisted of 777-946 amino acids, with calculated molecular weights from 88.70 to $107.50 \mathrm{kDa}$, and their isoelectric points (pI) ranged from 8.57 (CsRbohD) to 9.37 (CsRbohC). For the instability index (II), only one protein (CsRbohG) was predicted as stable. The grand average of the hydropathicity (GRAVY) value of all CsRboh proteins was less than zero, suggesting that these proteins were hydrophilic [21]. In addition, the in-silico subcellular localization prediction using Plant-mPLoc indicated that all CsRboh proteins were localized in the cell membrane [22]. 

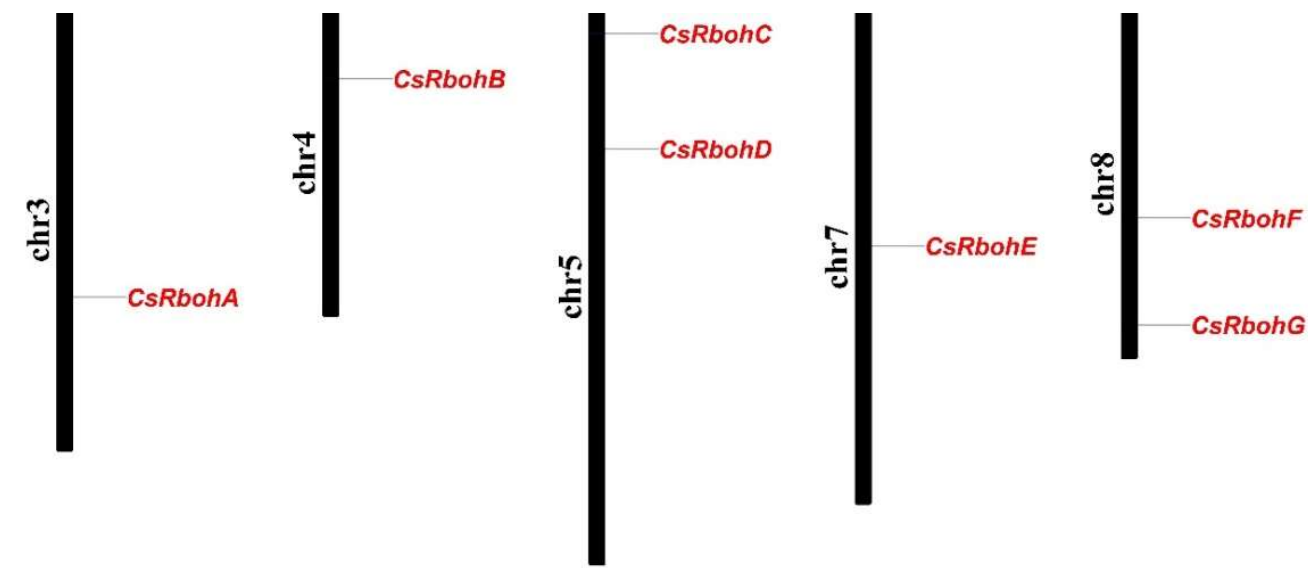

Figure 1. Chromosomal localization of the CsRboh genes. The chromosome numbers are indicated on the left of each chromosome.

Table 1. The details of the seven predicted CsRboh genes identified in the current study.

\begin{tabular}{|c|c|c|c|c|c|c|c|}
\hline $\begin{array}{l}\text { Gene } \\
\text { Name }\end{array}$ & $\begin{array}{l}\text { Locus } \\
\text { Number }\end{array}$ & $\begin{array}{c}\text { Amino } \\
\text { Acids } \\
\text { Length (aa) }\end{array}$ & $\begin{array}{c}\text { Molecular } \\
\text { Weight } \\
\text { (kDa) }\end{array}$ & $\begin{array}{c}\text { Isoelectric } \\
\text { Point }\end{array}$ & $\begin{array}{c}\text { Instability } \\
\text { Index }\end{array}$ & GRAVY & $\begin{array}{l}\text { Subcellular } \\
\text { Localization } \\
\text { Predicted }\end{array}$ \\
\hline CsRbohA & Cs3g14240 & 889 & 101.05 & 9.14 & 41.05 & -0.266 & Cell membrane \\
\hline CsRbohB & Cs4g06920 & 910 & 103.35 & 9.19 & 48.25 & -0.274 & Cell membrane \\
\hline CsRbohC & Cs5g02940 & 946 & 107.50 & 9.37 & 50.27 & -0.263 & Cell membrane \\
\hline CsRbohD & Cs5g11890 & 777 & 88.70 & 8.57 & 49.93 & -0.082 & Cell membrane \\
\hline CsRbohE & Cs7g19320 & 929 & 105.02 & 8.68 & 43.94 & -0.352 & Cell membrane \\
\hline CsRbohF & Cs8g12000 & 915 & 103.53 & 9.11 & 42.82 & -0.305 & Cell membrane \\
\hline CsRbohG & Cs8g17640 & 842 & 96.61 & 9.11 & 39.68 & -0.170 & Cell membrane \\
\hline
\end{tabular}

\subsection{Analysis of Domain Composition, Gene Structure, and Conserved Motifs of CsRbohs}

Through sequence alignment and Simple Modular Architecture Research Tool (SMART) analysis [23], we found that all the CsRboh protein sequences had four conserved domains, including NADPH_Ox and Ferric_reduct in the N-terminal region and FAD_binding_8 and NAD_binding_6 in the C-terminal region (Supplementary Figures S1 and S2). Furthermore, all the CsRboh protein sequences contained EF-hand domain and transmembrane regions except for CsRbohD. Among the ten motifs (Figure 2a), motif 10 was located in the NADPH_Ox domain region; motifs 2, 4, 7, and 9 consisted of the Ferric_reduct domain; motifs 1 and 5 were found in the FAD_binding_8 domain; and motifs 3, 5, and 8 constituted the NAD_binding_6 domain in all CsRboh proteins. Gene structure analysis revealed that the numbers of exons and introns among CsRboh genes varied between 12 and 14 and 11 and 13, respectively (Figure $2 b$ ). Notably, the size of CsRboh genes is generally determined by the size of introns. Overall, similar gene structures were found in some CsRboh genes clustered in the same group.

\subsection{Phylogenetic Analysis of CsRboh Genes}

To understand the evolutionary relationship among the Rbohs in Citrus, Arabidopsis, and rice, 26 Rboh predicted protein sequences were used to construct a phylogenetic tree (Figure 3). According to the phylogenetic tree, Rbohs were clustered into five groups, and the CsRbohs were distributed in each group. The CsRbohE and CsRbohF belonged to Group 1; CsRbohA was classified into Group 2; CsRbohD and CsRbohG were part of Group 3; CsRbohB was categorized into Group 4; CsRbohC was assigned into Group 5. 
a

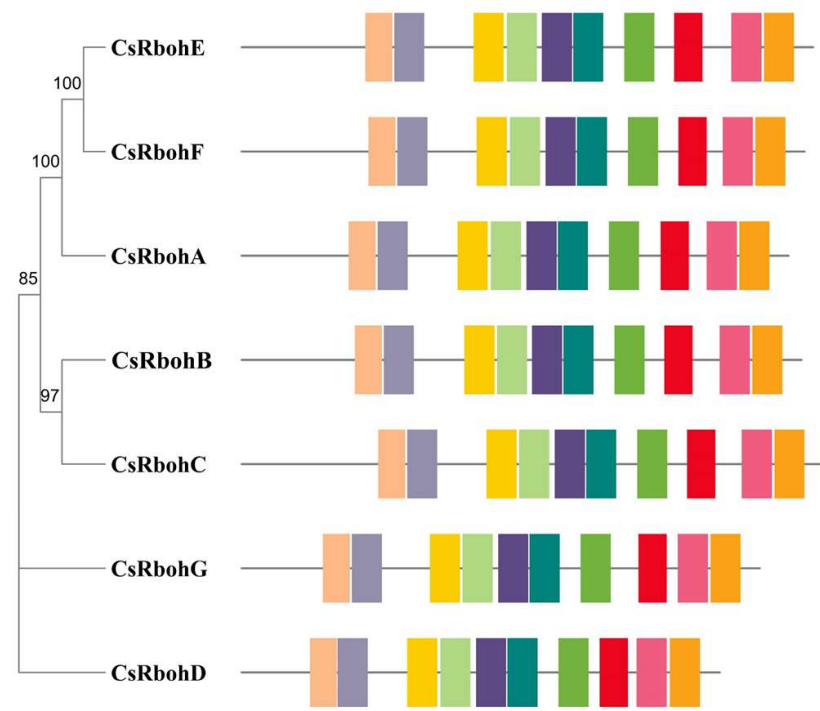

b

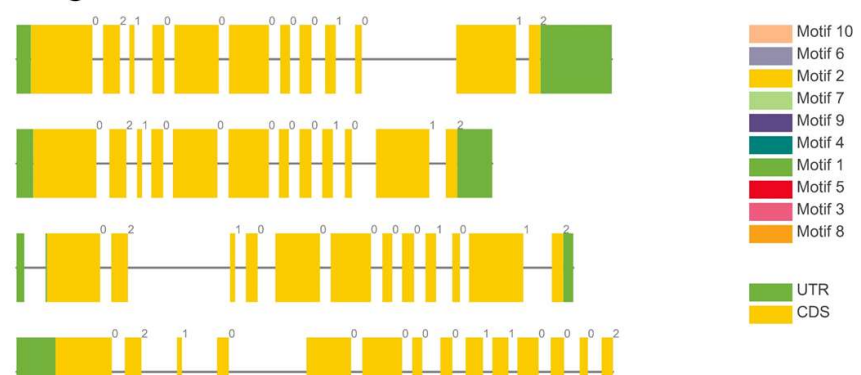

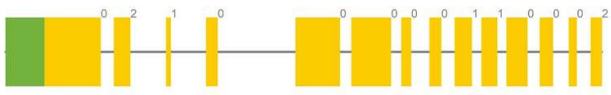

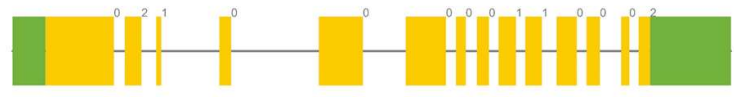

-

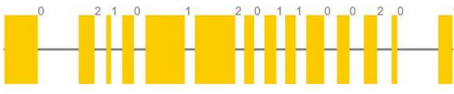

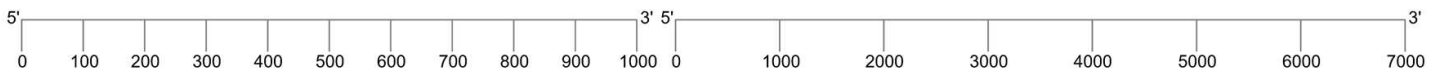

Figure 2. Evolutionary relationships, conserved motifs, and gene structures of CsRboh genes. The phylogenetic tree was constructed using MEGA-X by the Neighbour-Joining (NJ) method. (a) Ten types of conserved motifs in CsRboh proteins. (b) Gene structures of the CsRbohs. Exons, introns, and untranslated regions (UTRs) are indicated by yellow rectangles, gray lines, and green rectangles, respectively.

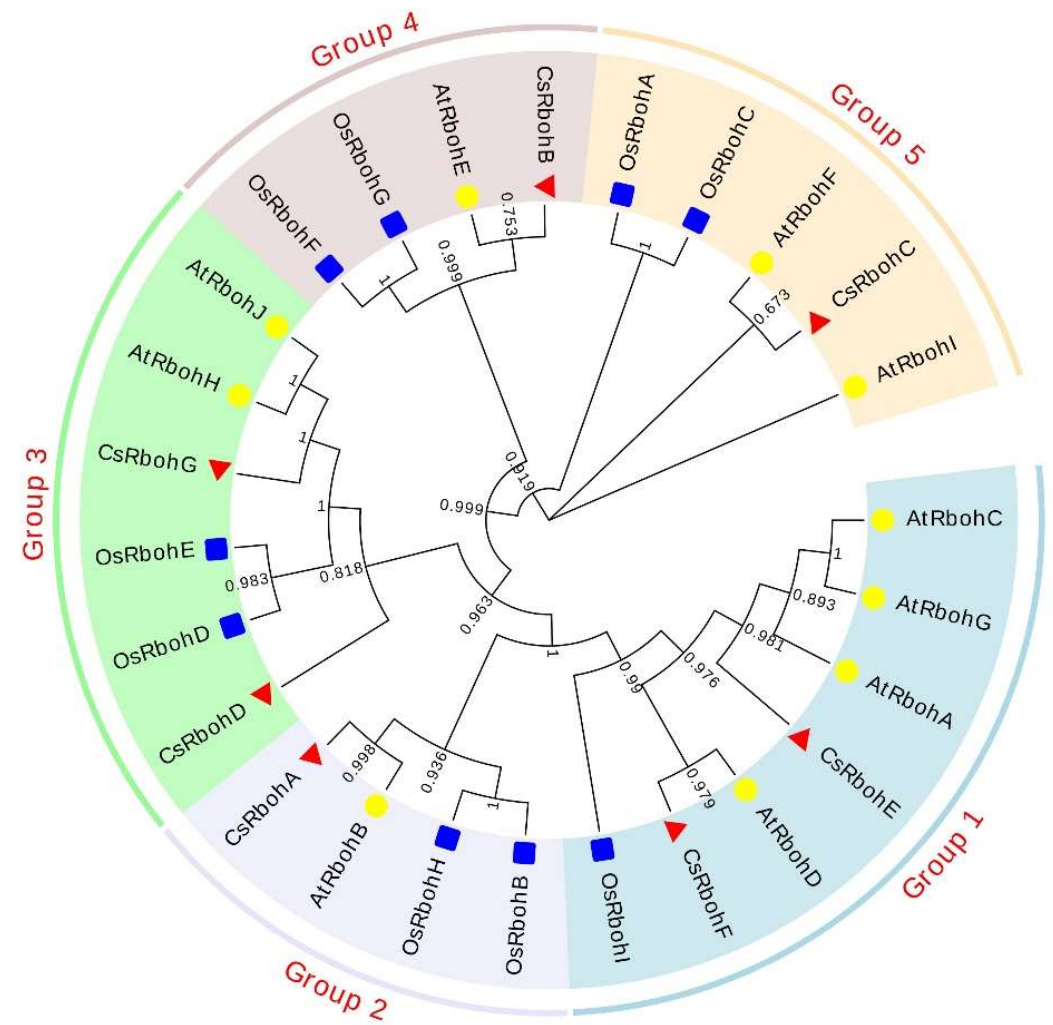

Figure 3. Phylogenetic tree of Rbohs in C. sinensis, rice, and Arabidopsis thaliana. The phylogenetic tree was constructed using MEGA-X by the NJ method with p-distance substitution model (gamma = 1) and 1000 bootstrap replicates. 


\subsection{Prediction of Cis-Acting Elements and miRNA Target Sites}

Cis-acting elements are known to play vital roles in the regulation of gene expression [24]. To better understand CsRboh gene function and transcriptional regulation mechanisms, the cis-acting elements in $1500 \mathrm{bp}$ upstream areas from the translation initiation site were predicted for all CsRboh genes using the PlantCARE database [25]. As presented in Figure 4, a total of fourteen representative cis-acting elements were selected, including five stress response elements (defense and stress responsiveness, low-temperature responsiveness, light responsiveness, anaerobic induction, and drought-inducibility), five hormone response elements (abscisic acid responsiveness, MeJA-responsiveness, auxinresponsiveness, gibberellin-responsiveness, and salicylic acid responsiveness), and four development regulation elements (seed-specific regulation, circadian control, differentiation of the palisade mesophyll cells, and meristem expression). Among these, the cis-acting elements of light responsiveness, MeJA-responsiveness, and abscisic acid responsiveness were highly enriched in the promoters of most CsRboh genes.

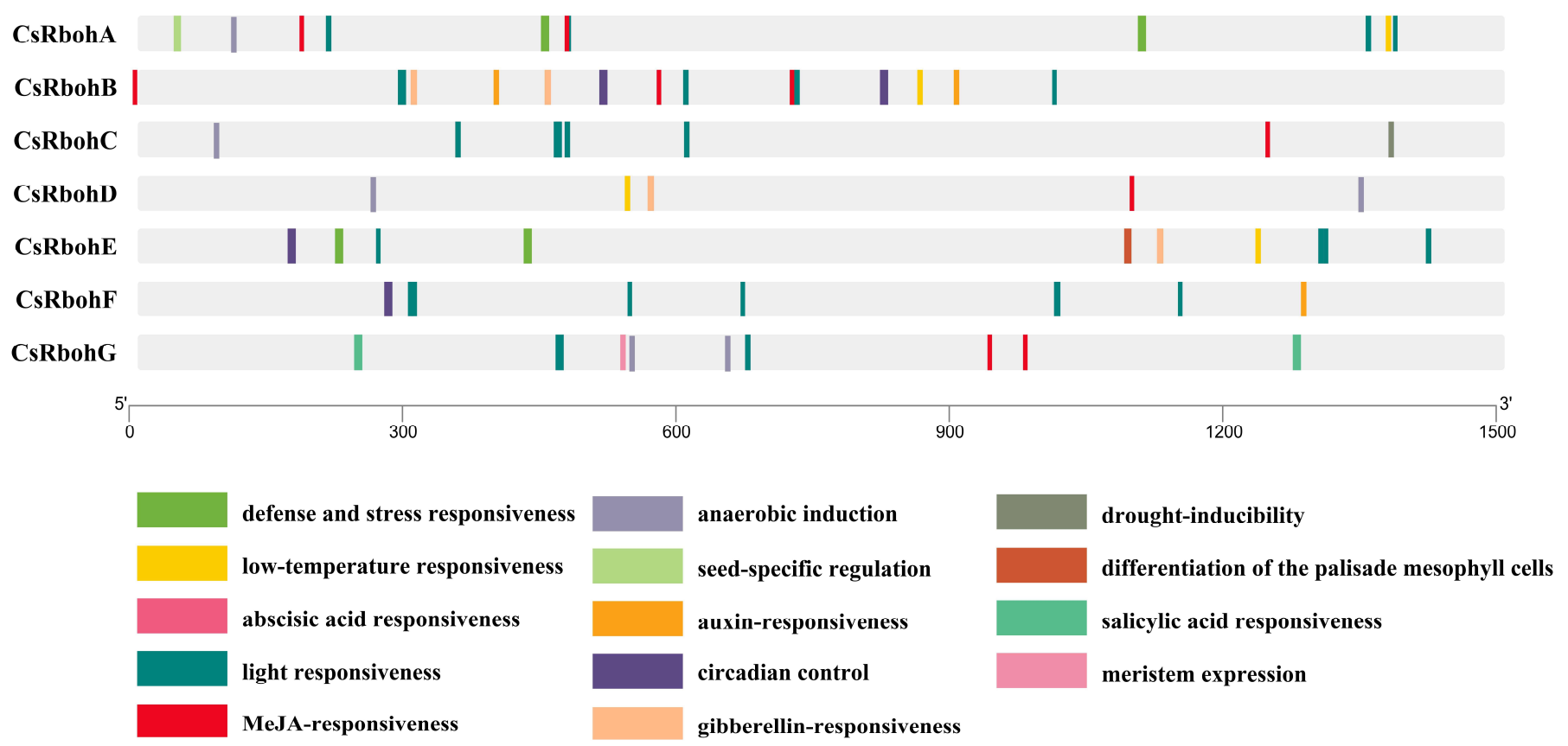

Figure 4. Prediction of Cis-acting elements in the CsRboh promoter regions. PlantCARE was used to identify the putative cis-acting element distribution in 1500 bp promoter sequences of 7 CsRbohs; cis-acting elements were divided into fourteen types.

All the miRNAs from Citrus were used as query sequences to predict the target sites on CsRboh genes by psRNATarget with stringent settings. CsRbohE was targeted by csi$\mathrm{miR} 164 \mathrm{a} / \mathrm{b} / \mathrm{c} / \mathrm{d}$ with sites in the NAD_binding_6 domain (Table 2).

Table 2. List of CsRboh genes with putative miRNA target sites.

\begin{tabular}{cccccc}
\hline Gene Name & $\begin{array}{c}\text { Locus } \\
\text { Number }\end{array}$ & $\begin{array}{c}\text { Predicted miRNA } \\
\text { Target Sites }\end{array}$ & miRNA Length & Expectation & UPE \\
\hline CsRbohE & Cs7g19320 & csi-miR164a & 21 & 3.5 & 23.655 \\
CsRbohE & Cs7g19320 & csi-miR164b & 21 & 3.5 & 23.655 \\
CsRbohE & Cs7g19320 & csi-miR164c & 21 & 3.5 & 23.655 \\
CsRbohE & Cs7g19320 & csi-miR164d & 21 & 3.5 & 23.655 \\
\hline
\end{tabular}

\subsection{Expression Analysis of CsRboh Genes in Different Tissues and Cold Stress Treatments}

To shed light on the possible function of CsRboh genes, the available RNA-seq data of the C. sinensis genome database (http:/ / citrus.hzau.edu.cn/ accessed on 10 May 2021) 
were analyzed [26]. The results showed these CsRboh genes had different tissue-specific expression patterns (Figure 5). CsRbohE was highly expressed in all examined tissues, whereas $C s R b o h G$ was expressed at very low levels. In addition, the expression pattern of CsRbohA and CsRbohD genes show similar profiles in the four tissues. To explore the response of CsRboh genes to cold stress in Citrus, the expression levels of CsRboh genes were detected after low-temperature treatment by qRT-PCR analysis. As shown in Figure 6, the expression levels of CsRbohA and CsRbohB were significantly down-regulated within $24 \mathrm{~h}$, while the transcript abundance of CsRbohD and CsRbohE was significantly increased at the $24 \mathrm{~h}$ time point, and CsRbohF was significantly upregulated at the $6 / 12 \mathrm{~h}$ time point. Furthermore, CsRbohC and CsRbohG gene expression did not differ significantly.

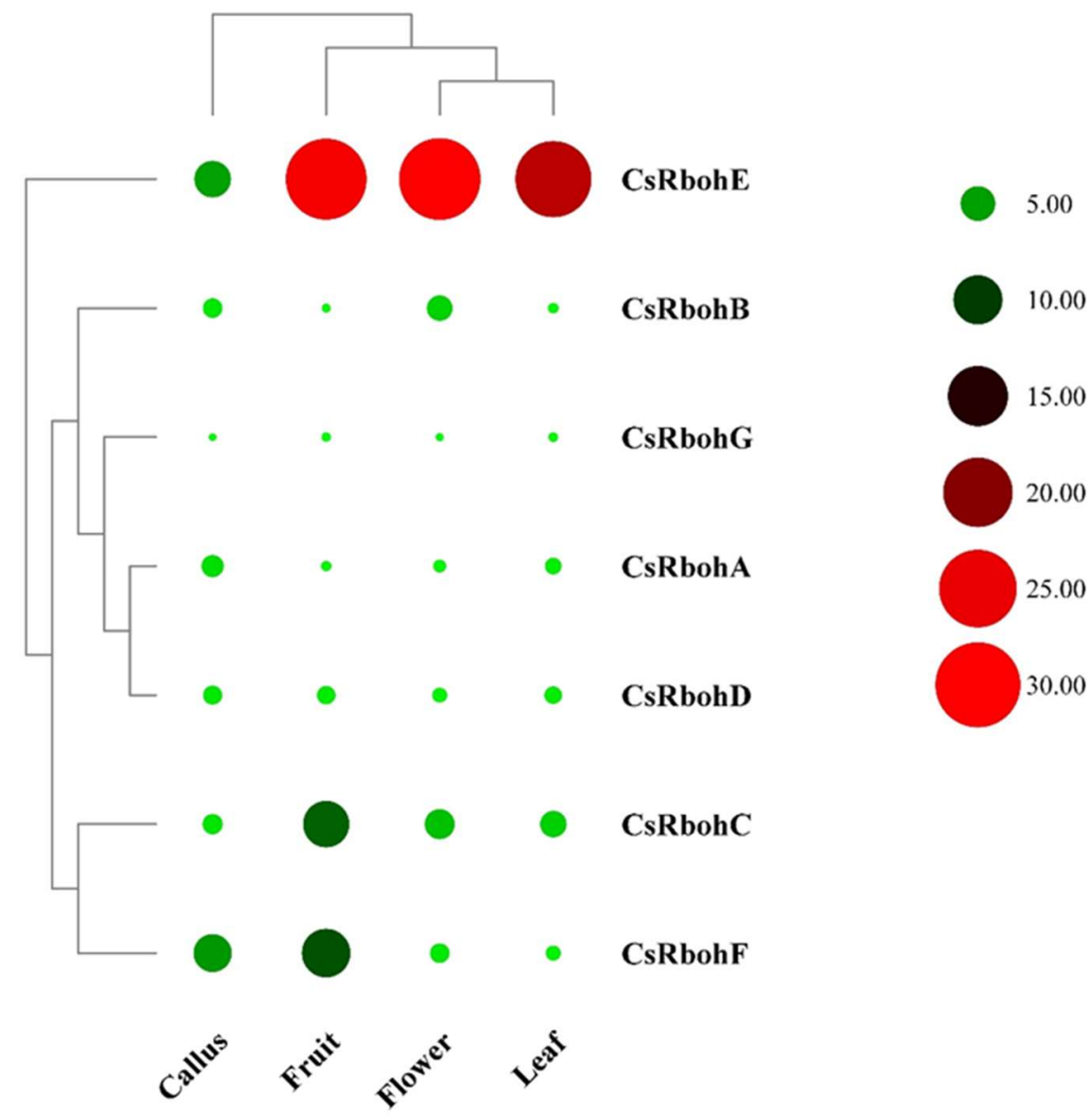

Figure 5. Expression patterns of the CsRboh genes in various tissues/organs using available RNAseq data. The colour scale and circle size are provided with the heat map to indicate the levels of differential expression. Red represents high expression level, green represents low expression level.

\subsection{Silencing of CsRbohD in Poncirus trifoliata by VIGS}

Given that $C s R b o h D$ was significantly induced by cold stress and a low-temperature responsive element was also present in the promoter of CsRbohD, subsequent analyses focused on this gene by VIGS in trifoliate oranges. CsRbohD transcript levels were repressed in ten VIGS plants, ranging from $22 \%$ to $77 \%$, compared to TRV empty vector control (Supplementary Figure S3). No obvious phenotypical differences were found between the TRV control plants and the VIGS lines without cold stress. However, when subjected to $-4{ }^{\circ} \mathrm{C}$ treatment for $12 \mathrm{~h}$, the VIGS lines exhibited more severe wilting, along with higher electrolyte leakage (EL) and malondialdehyde (MDA) contents, and weaker chlorophyll fluorescence and lower $\mathrm{F}_{\mathrm{v}} / \mathrm{F}_{\mathrm{m}}$ ratio in comparison with the TRV control plants (Figure 7a-e). Moreover, histochemical staining with nitroblue tetrazolium (NBT) and 3,3'-diaminobenzidine $(\mathrm{DAB})$, together with quantitative measurement, demonstrated that 
ROS accumulation was much greater in the VIGS lines than in the control (Figure $7 \mathrm{f}, \mathrm{h}$ ). By contrast, peroxidase (POD, EC 1.11.1.7) activities in the VIGS lines were significantly lower compared with those in control (Figure $7 \mathrm{~g}$ ). These results indicate that the silencing of CsRbohD in trifoliate orange compromised cold tolerance.
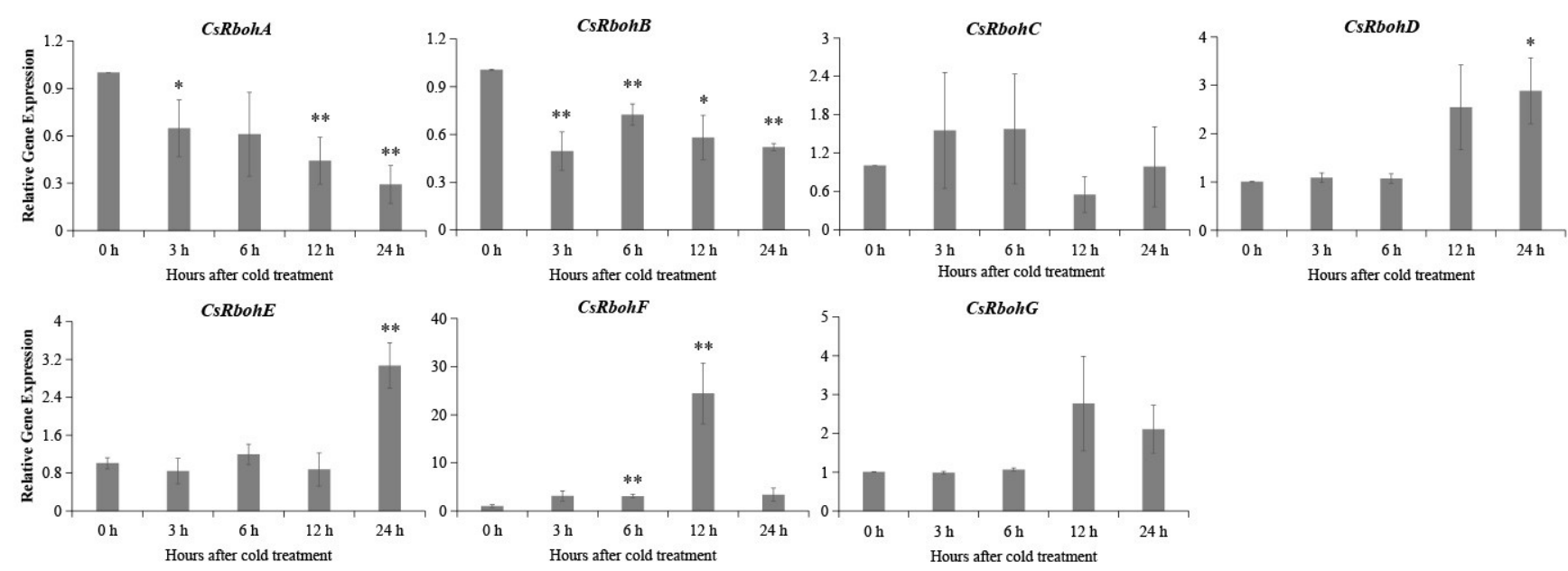

Figure 6. Expression patterns of the CsRboh genes under cold stress treatments. Asterisks indicate significant difference between control and cold treatment $\left({ }^{*} p<0.05,{ }^{* *} p<0.01\right)$.

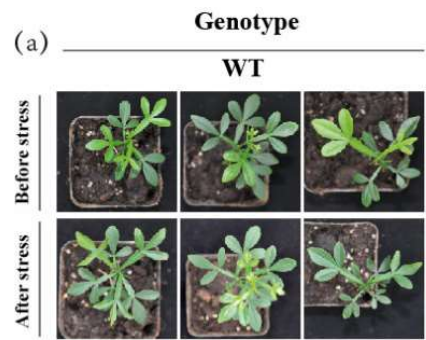

(b)
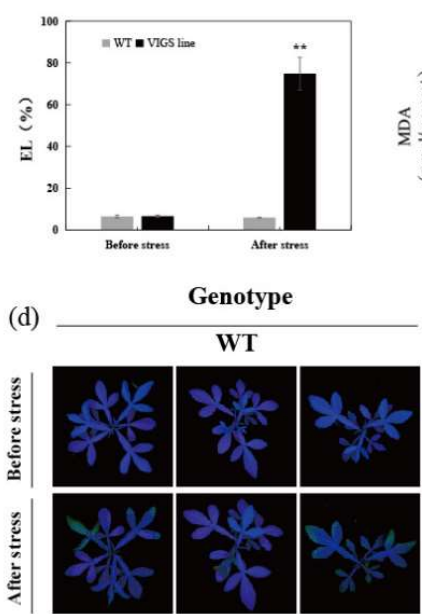

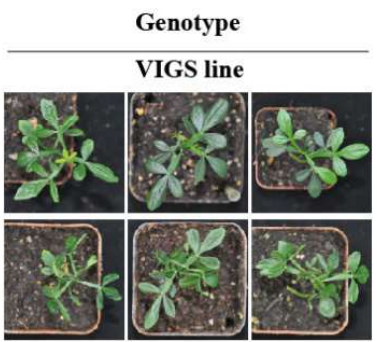

(c)

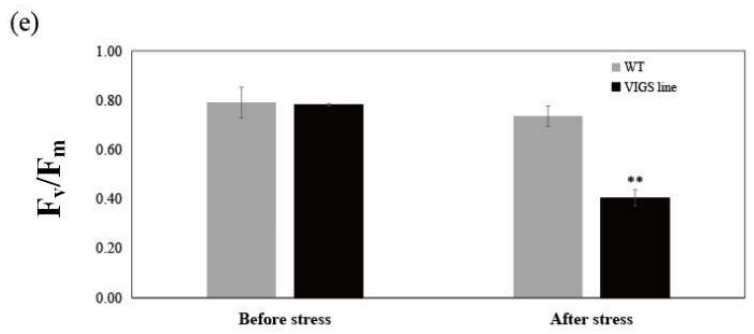

(f)
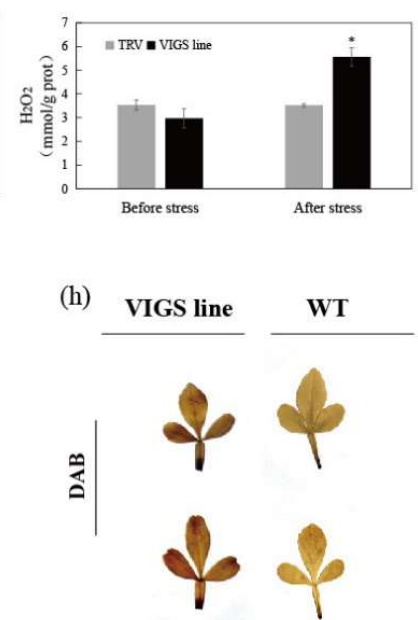

(g)
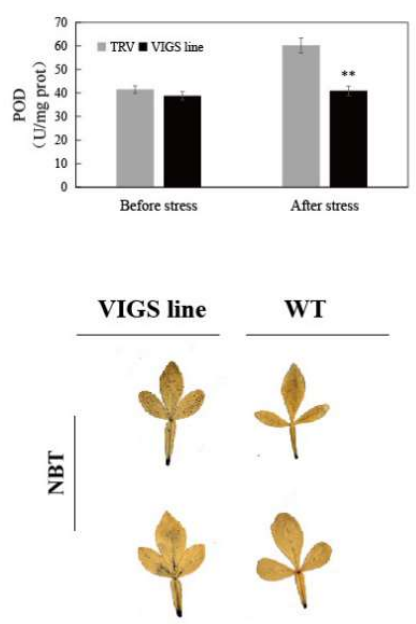

Figure 7. Silencing of CsRbohD by VIGS reduces cold tolerance in trifoliate orange. (a-g) Phenotype (a), EL (b), MDA level (c), Chlorophyll fluorescence imaging (d), $\mathrm{F}_{\mathrm{v}} / \mathrm{F}_{\mathrm{m}}$ ratio $(\mathbf{e}), \mathrm{H}_{2} \mathrm{O}_{2}$ content (f), and POD activity (g) of control plants (TRV) and VIGS plants (TRV-CsRbohD) before and after the freezing treatment $\left(12 \mathrm{~h}\right.$ at $\left.-4{ }^{\circ} \mathrm{C}\right)$. (h) DAB and NBT staining of the leaves of trifoliate orange under cold stress. Asterisks indicate significant difference between the WT and the VIGS lines $\left(^{*} p<0.05\right.$, ** $p<0.01$ ). 


\section{Discussion}

Rbohs are responsible for ROS generation and, thereby, are involved in regulating a diverse range of biological processes including development and stress responses in plants $[3,27]$. However, no comprehensive analysis of the Rboh gene family has been reported in Citrus. Here, we identified a total of seven Rboh genes from the Citrus sinensis genome (Table 1). The number of Rboh genes in Citrus is similar to previous reports of grapes [8], strawberries [12], jatropha [13], cassava [14], and pepper [16], but relatively small. Prediction programs for subcellular localization showed that the seven CsRboh proteins were localized in the cell membrane. The results are in agreement with those obtained in other studies $[10,13,14,16,28]$, suggesting Rbohs' potential role in the regulation of ROS generation. Notably, few Rboh proteins were predicted to localize in chloroplast thylakoid membranes or mitochondria. For instance, the FvRbohC, VvRbohB, VvRbohC2, VvRbohE, and $\mathrm{VvRbohH}_{\mathrm{v}}$ proteins were found in the chloroplast thylakoid membrane [8,12], and GaRboh9, GrRboh5, GhRboh2/15, and GbRboh2/15 were found in the mitochondria [29], indicating different cellular functions. Analysis of the domain composition, gene structure, and conserved motifs of CsRbohs revealed that members of the CsRboh gene family were relatively conserved during evolution (Figure 2, Supplementary Figures S1 and S2).

Phylogenetic analysis demonstrated that Rbohs of Citrus, Arabidopsis, and rice were clustered into five groups, consistent with earlier reports [12,16], and the CsRbohs showed a closer evolutionary relationship to Arabidopsis compared with those from rice (Figure 3). In addition, most genes within the same group had similar gene structures, implying that members of the same phylogenetic subgroup may have similar functions. The available RNA-seq data showed that CsRbohE, clustered in the same group 1 as the AtRbohD gene, was highly expressed in all four tissues (Figure 5). Again, the homologous gene AtrbohD was expressed in all tissues of Arabidopsis and was involved in many physiological processes such as stomatal closure, root formation, and systemic signaling in response to diverse stresses $[1,30]$. Therefore, we speculate that CsRbohE may have a similar function to AtRbohD. Nevertheless, further studies are necessary to confirm this point.

Analysis with PlantCARE showed that there were various cis-acting elements present in the promoters of the CsRboh genes (Figure 4) that could be grouped into three different functional categories, namely stress response elements, hormone response elements, and development regulation elements [25]. This kind of distribution pattern of cis-acting elements was also reported for many other plants, such as Arabidopsis, rice, and cotton [10,24]. Such results indicated that $R b o h$ genes function in multiple physiological processes, including development, biotic and abiotic stress responses, and hormone signaling. Moreover, it was surprising that $52.6 \%$ of cis-acting elements identified in the promoter regions of CsRbohs were related to stress response elements. Among them, the LTR motif (low-temperature responsive cis-acting element; CCGAAA) was found in the promoter regions of four CsRboh genes (CsRbohA, CsRbohB, CsRbohD, and CsRbohE). Thus, we investigated the responses of the CsRboh genes to low-temperature stress by qRT-PCR analysis (Figure 6). As expected, the four CsRboh genes were differentially expressed after low-temperature treatment. Interestingly, CsRbohA-B were up-regulated while CsRbohD-E were down-regulated, indicating their potential important roles in defense against cold stress as positive or negative regulators. In model plant Arabidopsis thaliana, the low-temperature responsive cis-acting element was also observed in the promoters of all AtRboh genes except for AtRbohD-E, and cold stress caused the up- and down-regulation of these AtRboh genes [24]. Similarly, three rice $R b o h$ genes (OsRbohD, OsRbohH, and OsRbohI) were up-regulated after cold stress. The promoter regions of these genes contained low-temperature responsive cis-acting elements [24]. Collectively, the low-temperature responsive cis-acting elements may be involved in cold stress-induced Rboh gene expression.

A class of small noncoding RNAs, miRNAs, have been shown to play a key role in negatively regulating gene expression at the post transcriptional level [31]. Previous research has indicated that miR164 is involved in many biological processes including multiple stress responses and regulating shoot apical meristems, lateral root development, 
cell death, and fruit ripening [32-36]. Our results revealed that csi-miR164 may target the CsRbohE gene. This prediction would need to be further verified by experiments.

It is well known that Rbohs have emerged as key hubs for ROS signaling in plants [3,30]. So far, more than 56 members have been fully characterized in function [3]. However, the regulatory functions of Rbohs in response to cold stress have not been reported. Here, we silenced the CsRbohD gene using VIGS in trifoliate oranges, and found that silencing of CsRbohD greatly compromised cold tolerance (Figure 7). Considering the fact that high levels of ROS and decreased activities of antioxidant enzymes (POD) were detected in the VIGS lines, we hypothesize that a possible CsRbohD-ROS signal pathway induces ROS-scavenging enzyme genes under cold stress conditions.

\section{Materials and Methods}

\subsection{Genome-Wide Identification of Citrus sinensis Rboh Family Genes}

Both the Citrus genome and protein sequences were retrieved from databases (C. sinensis genome from http:/ / citrus.hzau.edu.cn/ accessed on 10 May 2021). To identify Citrus Rboh genes, BLASTP was performed using ten Arabidopsis Rboh protein sequences retrieved from The Arabidopsis Information Resource (TAIR, https:/ /www.arabidopsis.org/ accessed on 10 May 2021), and the Hidden Markov Model (HMM) profile of the respiratory burst NADPH oxidase domain (PF08414), ferric reductase like transmembrane component domain (PF01794), FAD-binding domain (PF08022), and ferric reductase NAD binding domain (PF08030) downloaded from the pfam (http:/ / pfam.xfam.org/ accessed on 10 May 2021) as queries. All the putative CsRbohs were further confirmed through the Pfam database and the SMART database for conserved domains. Based to their chromosomal location, we named these genes CsRbohA-G. The chromosomal location map of CsRboh genes was displayed by the TBtool software (https://github.com/CJ-Chen/TBtools/ accessed on 10 May 2021) [37].

\subsection{Determination of Protein Properties, Subcellular Location, Gene Structure, and Conserved Motifs}

Protein properties including molecular weight, isoelectric point, instability index, and the grand average of hydropathicity were predicted using the ExPASy-ProtParam tool (http:/ / web.expasy.org/protparam/ accessed on 12 May 2021). The subcellular localizations of CsRboh proteins were analyzed using Plant-mPLoc (http:/ / www.csbio.sjtu.edu.cn/ bioinf / plant-multi / accessed on 12 May 2021). For the exon-intron structure determination, the coding sequences (CDS) of CsRboh genes were aligned with its genomic DNA sequence using TBtools software. The conserved motifs of CsRboh proteins were predicted using MEME server v 5.4.1 (http://meme-suite.org/tools/meme/ accessed on 12 May 2021) with default parameters except for the motif number set to 10 [38].

\subsection{Multiple Sequence Alignment and Phylogenetic Analysis}

Multiple alignments of the CsRboh amino acid sequences were performed using ClustalW software. The Arabidopsis and rice sequence data used in this study were collected from the National Center for Biotechnology Information (NCBI) (http:/ / www.ncbi.nlm. nih.gov / accessed on 13 May 2021). The phylogenetic tree was created using MEGA X [39] (https:/ / www.megasoftware.net/ accessed on 10 June 2020) by Neighbor-joining algorithm with boot strap replication of 1000 times.

\subsection{Identification of Cis-Acting Elements and Prediction of miRNA Target Sites}

The $1.5 \mathrm{~kb}$ region upstream of the transcription start site of each CsRboh gene was extracted from the $C$. sinensis genome sequence and used to predict cis-acting elements by PlantCARE [25] (http:/ / bioinformatics.psb.ugent.be/webtools/plantcare/html/ accessed on 20 May 2021). All known Citrus microRNA sequences were downloaded from miRBase database (Release 22.1) (http:/ / www.mirbase.org/index.shtml accessed on 17 June 2021). CsRbohs were analyzed for the presence of miRNA target sites using psRNATarget server 
(http: / / plantgrn.noble.org/psRNATarget/ accessed on 17 June 2021) [40] with stringent parameters (to avoid false positives, maximum expectation values were set 3.5; all other parameters were used in the default setting).

\subsection{Expression Patterns of CsRboh Genes}

mRNA-seq data for different Citrus tissues were downloaded from the sweet orange genome annotation database (http: / / citrus.hzau.edu.cn/ accessed on 10 May 2021) and calculated as retrieved fragments per kilobase of transcript per million fragments mapped (FPKM) values for CsRbohs.

\subsection{Plant Materials and Cold Treatments}

C. sinensis (Valencia sweet orange) seeds were sterilized in sodium hypochlorite (10\%) for $20 \mathrm{~min}$ and washed with distilled water three times, followed by sowing on MT medium added with $30 \mathrm{~g} \mathrm{~L}^{-1}$ sucrose. For cold treatments, 6-week-old seedlings were incubated at $4{ }^{\circ} \mathrm{C}$ for $0,3,6,12$, and $24 \mathrm{~h}$, after which the leaves were harvested into liquid nitrogen and stored at $-80^{\circ} \mathrm{C}$ until RNA extraction. For each experiment, three biological replicates were performed, and the leaves from five seedlings were collected for each replicate.

\subsection{RNA Extraction and Quantitative Real-Time PCR Analysis}

Total RNA was extracted using Trizol reagent (TaKaRa, Dalian, China) according to the manufacturer's instructions. The quality of the RNA was verified by gel electrophoresis and Bioanalyzer (Agilent2100). The first strand complementary DNA (cDNA) was synthesized using TransScript Reverse transcriptase (TaKaRa, Dalian, China) according to the manufacturer's instructions. The expression of the gene encoding $\beta$-actin was used as an internal expression control. The gene-specific primers used for the qRT-PCR were listed in Supplementary Table S1. QRT-PCR was performed with CFX96 real-time PCR machine (BIO-RAD, Berkeley, CA, USA). The reaction system and procedure of qPCR were performed according to the procedures previously described [41]. The $2^{-\Delta \Delta C T} \mathrm{Ct}$ method was used to calculate the relative gene expression level across the samples [42].

\subsection{Generation of VIGS Plants}

VIGS-mediated suppression of CsRbohD was conducted according to previous protocols [43]. A $420 \mathrm{bp}$ fragment of CsRbohD was amplified and inserted into the SmaI and BamHI sites of tobacco rattle virus-based vector 2 (TRV2) to obtain the pTRV2-CsRbohD construct. The vectors pTRV1, pTRV2, and pTRV2-CsRbohD were introduced into Agrobacterium tumefaciens strain GV3101 by heat shock. The bacterial solutions were used to infiltrate trifoliate orange germinating seeds with emerging shoots (around $2 \mathrm{~cm}$ ) as previously described [43]. After the infiltration, the seedlings were then rinsed with water, dried on filter paper, and transplanted to a controlled growth chamber (Percival, IA, USA) for approximately 1 month. Fully expanded leaves were collected from each plant and subjected to genomic PCR and qRT-PCR analyses, and the seedlings that exhibited silencing of CsRbohD were used for further analysis.

\subsection{Cold Tolerance Assays, Physiological Measurements and Histochemical Staining}

For cold tolerance assay, about 1-month-old VIGS plants and WT plants were exposed to $-4{ }^{\circ} \mathrm{C}$ for $12 \mathrm{~h}$. Growth performance and chlorophyll fluorescence imaging of the plants were analyzed, while leaves were harvested for physiological analyses or histochemical staining. The MDA and $\mathrm{H}_{2} \mathrm{O}_{2}$ levels and POD activities were determined using corresponding assay kits (Nanjing Jiancheng Bioengineering Institute, China). MDA assay was performed using the thiobarbituric acid method and calculated by the absorbance of TBA reactive substances at $532 \mathrm{~nm} . \mathrm{H}_{2} \mathrm{O}_{2}$ assay was based on the oxidative polymerization of molybdic acid into a complex compound which can be determined at $405 \mathrm{~nm}$. The MDA or $\mathrm{H}_{2} \mathrm{O}_{2}$ contents were expressed as nmol mg $\mathrm{mg}^{-1}$ protein or $\mathrm{mmol} \mathrm{g}^{-1}$ protein, respectively. One unit of POD activity was defined as the amount of enzyme required to the formation of 
$1 \mu \mathrm{mol}$ product per minute. EL was calculated based on previous reports [44], and $\mathrm{F}_{\mathrm{v}} / \mathrm{F}_{\mathrm{m}}$ ratios were measured using Imaging WinGegE software. Furthermore, histochemical staining was used for the in vivo detection of $\mathrm{H}_{2} \mathrm{O}_{2}$ and $\mathrm{O}_{2}{ }^{-}$with DAB and NBT, respectively [45].

\subsection{Statistical Analysis}

Cold treatment was repeated at least three times, with three replicates for each line and time point. All data, expressed as mean \pm SD, were analyzed by SPSS 25.0 software (IBM Corp., Armonk, NY, USA). Analysis of variance (ANOVA) was used to compare the statistical difference based on Fisher's least significant difference test at the significance levels of $p<0.05\left(^{*}\right)$, and $p<0.01\left(^{* *}\right)$.

\section{Conclusions}

In our study, seven Rboh genes (CsRbohA CsRbohG) were identified in the genome of $C$. sinensis and clustered into five groups. Gene structure, motif distribution pattern, and cis-acting element analysis indicated the conservation and divergence of CsRboh genes. Csi-miR164 was predicted to target the CsRbohE gene. Five CsRboh genes were responsive to low-temperature treatment. Functional and physiological analyses suggested that the CsRbohD gene is involved in response to cold stress. As a whole, our results lay a foundation for further functional characterization of CsRbohs, and provide key candidate proteins for genetic improvement of Citrus.

Supplementary Materials: The following figures and a table are available online at https://www. mdpi.com/article/10.3390/ijms23020648/s1, Figure S1: Alignment of the CsRboh protein sequences, Figure S2: Domain compositions of CsRboh proteins by SMART analysis, Figure S3: Molecular characterization of the VIGS plants by genomic PCR and qRT-PCR, Table S1: Primers used in this study.

Author Contributions: Conceptualization, X.X.; methodology, Y.Z. (Yueliang Zhang) and Y.Z. (Yiwu Zhang); software, L.C. and X.X.; validation, Y.Z. (Yueliang Zhang), C.L., L.L. and Y.Z. (Yiwu Zhang); resources, J.L.; data curation, Y.Z. (Yueliang Zhang) and L.L.; writing - original draft preparation, X.X., W.K. and J.L.; writing - review and editing, X.X.; supervision, X.X. and J.L.; project administration, X.X.; funding acquisition, X.X. All authors have read and agreed to the published version of the manuscript.

Funding: This research was funded by the National Key Research and Development Program of China (2018YFD1000107); Agricultural Science \& Technology Independent Innovation Fund of Jiangsu Province [CX(21)3024]; and the Natural Science Foundation of Jiangsu Province, China (BK20181454).

Institutional Review Board Statement: Not applicable.

Informed Consent Statement: Not applicable.

Data Availability Statement: Not applicable.

Conflicts of Interest: The authors declare no conflict of interest.

\section{References}

1. Suzuki, N.; Miller, G.; Morales, J.; Shulaev, V.; Torres, M.A.; Mittler, R. Respiratory burst oxidases: The engines of ROS signaling. Curr. Opin. Plant Biol. 2011, 14, 691-699. [CrossRef]

2. Oda, T.; Hashimoto, H.; Kuwabara, N.; Akashi, S.; Hayashi, K.; Kojima, C.; Wong, H.L.; Kawasaki, T.; Shimamoto, K.; Sato, M.; et al. Structure of the N-terminal Regulatory Domain of a Plant NADPH Oxidase and Its Functional Implications. J. Biol. Chem. 2010, 285, 1435-1445. [CrossRef]

3. Hu, C.-H.; Wang, P.-Q.; Zhang, P.-P.; Nie, X.-M.; Li, B.-B.; Tai, L.; Liu, W.-T.; Li, W.-Q.; Chen, K.-M. NADPH Oxidases: The Vital Performers and Center Hubs during Plant Growth and Signaling. Cells 2020, 9, 437. [CrossRef]

4. Wang, W.; Chen, D.; Zhang, X.; Liu, D.; Cheng, Y.; Shen, F. Role of plant respiratory burst oxidase homologs in stress responses. Free Radic. Res. 2018, 52, 826-839. [CrossRef]

5. Groom, Q.J.; Torres, M.A.; Fordham-Skelton, A.P.; Hammond-Kosack, K.E.; Robinson, N.J.; Jones, J.D. rbohA, a rice homologue of the mammalian gp91phox respiratory burst oxidase gene. Plant J. 1996, 10, 515-522. [CrossRef]

6. Sagi, M.; Fluhr, R. Production of Reactive Oxygen Species by Plant NADPH Oxidases. Plant Physiol. 2006, 141, 336-340. [CrossRef] [PubMed] 
7. Wang, G.-F.; Li, W.-Q.; Wu, G.-L.; Zhou, C.-Y.; Chen, K.-M. Characterization of Rice NADPH Oxidase Genes and Their Expression under Various Environmental Conditions. Int. J. Mol. Sci. 2013, 14, 9440-9458. [CrossRef]

8. Cheng, C.; Xu, X.; Gao, M.; Li, J.; Guo, C.; Song, J.; Wang, X. Genome-Wide Analysis of Respiratory Burst Oxidase Homologs in Grape (Vitis vinifera L.). Int. J. Mol. Sci. 2013, 14, 24169-24186. [CrossRef]

9. Yu, S.; Kakar, K.U.; Yang, Z.; Nawaz, Z.; Lin, S.; Guo, Y.; Ren, X.-L.; Baloch, A.A.; Han, D. Systematic study of the stress-responsive Rboh gene family in Nicotiana tabacum: Genome-wide identification, evolution and role in disease resistance. Genomics 2020, 112, 1404-1418. [CrossRef]

10. Wang, W.; Chen, D.; Liu, D.; Cheng, Y.; Zhang, X.; Song, L.; Hu, M.; Dong, J.; Shen, F. Comprehensive analysis of the Gossypium hirsutum L. respiratory burst oxidase homolog (Ghrboh) gene family. BMC Genom. 2020, 21, 91. [CrossRef] [PubMed]

11. Cepauskas, D.; Miliute, I.; Staniene, G.; Gelvonauskiene, D.; Stanys, V.; Jesaitis, A.; Baniulis, D. Characterization of apple NADPH oxidase genes and their expression associated with oxidative stress in shoot culture in vitro. Plant Cell Tissue Organ Cult. (PCTOC) 2015, 124, 621-633. [CrossRef]

12. Zhang, Y.; Li, Y.; He, Y.; Hu, W.; Zhang, Y.; Wang, X.; Tang, H. Identification of NADPH oxidase family members associated with cold stress in strawberry. FEBS Open Bio. 2018, 8, 593-605. [CrossRef] [PubMed]

13. Zhao, Y.; Zou, Z. Genomics analysis of genes encoding respiratory burst oxidase homologs (RBOHs) in jatropha and the comparison with castor bean. PeerJ 2019, 7, e7263. [CrossRef] [PubMed]

14. Huang, S.; Tang, Z.; Zhao, R.; Hong, Y.; Zhu, S.; Fan, R.; Ding, K.; Cao, M.; Luo, K.; Geng, M.; et al. Genome-wide identification of cassava MeRboh genes and functional analysis in Arabidopsis. Plant Physiol. Biochem. 2021, 167, 296-308. [CrossRef]

15. Li, D.; Wu, D.; Li, S.; Dai, Y.; Cao, Y. Evolutionary and functional analysis of the plant-specific NADPH oxidase gene family in Brassica rapa L. R. Soc. Open Sci. 2019, 6, 181727. [CrossRef] [PubMed]

16. Zhang, J.; Xie, Y.; Ali, B.; Ahmed, W.; Tang, Y.; Li, H. Genome-wide Identification, Classification, Evolutionary Expansion and Expression of Rboh Family Genes in Pepper (Capsicum annuum L.). Trop. Plant Biol. 2021, 14, 251-266. [CrossRef]

17. Wong, H.L.; Pinontoan, R.; Hayashi, K.; Tabata, R.; Yaeno, T.; Hasegawa, K.; Kojima, C.; Yoshioka, H.; Iba, K.; Kawasaki, T.; et al Regulation of Rice NADPH Oxidase by Binding of Rac GTPase to Its N-Terminal Extension. Plant Cell 2007, 19, $4022-4034$. [CrossRef]

18. Liu, J.; Lu, H.; Wan, Q.; Qi, W.; Shao, H. Genome-wide analysis and expression profiling of respiratory burst oxidase homologue gene family in Glycine max. Environ. Exp. Bot. 2018, 161, 344-356. [CrossRef]

19. Mohammadrezakhani, S.; Rezanejad, F.; Hajilou, J. Effect of putrescine and proline on proflies of GABA, antioxidant activities in leaves of three Citrus species in response to low temperature stress. J. Plant Biochem. Biotechnol. 2021, 1-9. [CrossRef]

20. Cheng, X.; Li, G.; Manzoor, M.A.; Wang, H.; Abdullah, M.; Su, X.; Zhang, J.; Jiang, T.; Jin, Q.; Cai, Y.; et al. In Silico Genome-Wide Analysis of Respiratory Burst Oxidase Homolog (RBOH) Family Genes in Five Fruit-Producing Trees, and Potential Functional Analysis on Lignification of Stone Cells in Chinese White Pear. Cells 2019, 8, 520. [CrossRef]

21. Gasteiger, E.; Hoogland, C.; Gattiker, A.; Wilkins, M.R.; Appel, R.D.; Bairoch, A. Protein identification and analysis tools on the ExPASy server. In The Proteomics Protocols Handbook; Springer: Totowa, NJ, USA, 2005; pp. 571-607. [CrossRef]

22. Chou, K.-C.; Shen, H.-B. Plant-mPLoc: A Top-Down Strategy to Augment the Power for Predicting Plant Protein Subcellular Localization. PLoS ONE 2010, 5, e11335. [CrossRef]

23. Letunic, I.; Khedkar, S.; Bork, P. SMART: Recent updates, new developments and status in 2020. Nucleic Acids Res. 2020, 49, D458-D460. [CrossRef]

24. Kaur, G.; Pati, P.K. Analysis of cis-acting regulatory elements of Respiratory burst oxidase homolog ( Rboh ) gene families in Arabidopsis and rice provides clues for their diverse functions. Comput. Biol. Chem. 2016, 62, 104-118. [CrossRef] [PubMed]

25. Lescot, M.; Déhais, P.; Thijs, G.; Marchal, K.; Moreau, Y.; Van de Peer, Y.; Rouzé, P.; Rombauts, S. PlantCARE, a database of plant cis-acting regulatory elements and a portal to tools for in silico analysis of promoter sequences. Nucleic Acids Res. 2002, 30, 325-327. [CrossRef]

26. Xu, Q.; Chen, L.-L.; Ruan, X.; Chen, D.; Zhu, A.; Chen, C.; Bertrand, D.; Jiao, W.-B.; Hao, B.-H.; Lyon, M.P.; et al. The draft genome of sweet orange (Citrus sinensis). Nat. Genet. 2012, 45, 59-66. [CrossRef]

27. Chapman, J.M.; Muhlemann, J.K.; Gayomba, S.R.; Muday, G.K. RBOH-Dependent ROS Synthesis and ROS Scavenging by Plant Specialized Metabolites To Modulate Plant Development and Stress Responses. Chem. Res. Toxicol. 2019, 32, 370-396. [CrossRef]

28. Cheng, C.; Che, Q.; Su, S.; Liu, Y.; Wang, Y.; Xu, X. Genome-wide identification and characterization of Respiratory Burst Oxidase Homolog genes in six Rosaceae species and an analysis of their effects on adventitious rooting in apple. PLoS ONE 2020, 15, e0239705. [CrossRef]

29. Chang, Y.; Li, B.; Shi, Q.; Geng, R.; Geng, S.; Liu, J.; Zhang, Y.; Cai, Y. Comprehensive Analysis of Respiratory Burst Oxidase Homologs (Rboh) Gene Family and Function of GbRboh5/18 on Verticillium Wilt Resistance in Gossypium barbadense. Front. Genet. 2020, 11. [CrossRef] [PubMed]

30. Sun, L.R.; Zhao, Z.J.; Hao, F.S. NADPH oxidases, essential players of hormone signalings in plant development and response to stresses. Plant Signal. Behav. 2019, 14, 1657343. [CrossRef] [PubMed]

31. Pagano, L.; Rossi, R.; Paesano, L.; Marmiroli, N.; Marmiroli, M. miRNA regulation and stress adaptation in plants. Environ. Exp. Bot. 2021, 184, 104369. [CrossRef]

32. Wang, W.; Wang, J.; Wu, Y.; Li, D.; Allan, A.C.; Yin, X. Genome-wide analysis of coding and non-coding RNA reveals a conserved miR164- NAC regulatory pathway for fruit ripening. New Phytol. 2019, 225, 1618-1634. [CrossRef] 
33. Shan, T.; Fu, R.; Xie, Y.; Chen, Q.; Wang, Y.; Li, Z.; Song, X.; Li, P.; Wang, B. Regulatory Mechanism of Maize (Zea mays L.) miR164 in Salt Stress Response. Russ. J. Genet. 2020, 56, 835-842. [CrossRef]

34. Hu, G.; Lei, Y.; Liu, J.; Hao, M.; Zhang, Z.; Tang, Y.; Chen, A.; Wu, J. The ghr-miR164 and GhNAC100 modulate cotton plant resistance against Verticillium dahlia. Plant Sci. 2020, 293, 110438. [CrossRef]

35. Fang, Y.; Xie, K.; Xiong, L. Conserved miR164-targeted NAC genes negatively regulate drought resistance in rice. J. Exp. Bot. 2014, 65, 2119-2135. [CrossRef]

36. Kim, J.H.; Woo, H.R.; Kim, J.; Lim, P.O.; Lee, I.C.; Choi, S.H.; Hwang, D.; Gil Nam, H. Trifurcate Feed-Forward Regulation of Age-Dependent Cell Death Involving miR164 in Arabidopsis. Science 2009, 323, 1053-1057. [CrossRef]

37. Chen, C.; Chen, H.; Zhang, Y.; Thomas, H.R.; Frank, M.H.; He, Y.; Xia, R. TBtools: An Integrative Toolkit Developed for Interactive Analyses of Big Biological Data. Mol. Plant 2020, 13, 1194-1202. [CrossRef]

38. Bailey, T.L.; Boden, M.; Buske, F.A.; Frith, M.; Grant, C.E.; Clementi, L.; Ren, J.; Li, W.W.; Noble, W.S. MEME SUITE: Tools for motif discovery and searching. Nucleic Acids Res. 2009, 37 (Suppl. 2), w202-w208. [CrossRef]

39. Kumar, S.; Stecher, G.; Tamura, K. MEGA7: Molecular Evolutionary Genetics Analysis Version 7.0 for Bigger Datasets. Mol. Biol. Evol. 2016, 33, 1870-1874. [CrossRef]

40. Dai, X.; Zhao, P.X. psRNATarget: A plant small RNA target analysis server. Nucleic Acids Res. 2011, 39, W155-W159. [CrossRef] [PubMed]

41. Xu, X.; Zhou, Y.; Wang, B.; Ding, L.; Wang, Y.; Luo, L.; Zhang, Y.; Kong, W. Genome-wide identification and characterization of laccase gene family in Citrus sinensis. Gene 2018, 689, 114-123. [CrossRef]

42. Livak, K.J.; Schmittgen, T.D. Analysis of Relative Gene Expression Data Using Real-Time Quantitative PCR and the $2^{-\Delta \Delta C T}$ Method. Methods 2001, 25, 402-408. [CrossRef]

43. Dai, W.; Wang, M.; Gong, X.; Liu, J. The transcription factor Fc WRKY 40 of Fortunella crassifolia functions positively in salt tolerance through modulation of ion homeostasis and proline biosynthesis by directly regulating SOS 2 and P5 CS 1 homologs. New Phytol. 2018, 219, 972-989. [CrossRef]

44. Shi, J.; Fu, X.-Z.; Peng, T.; Huang, X.-S.; Fan, Q.-J.; Liu, J.-H. Spermine pretreatment confers dehydration tolerance of citrus in vitro plants via modulation of antioxidative capacity and stomatal response. Tree Physiol. 2010, 30, 914-922. [CrossRef]

45. Huang, X.-S.; Wang, W.; Zhang, Q.; Liu, J.-H. A Basic Helix-Loop-Helix Transcription Factor, PtrbHLH, of Poncirus trifoliata Confers Cold Tolerance and Modulates Peroxidase-Mediated Scavenging of Hydrogen Peroxide. Plant Physiol. 2013, 162, 1178-1194. [CrossRef] 